\title{
Patterning polyethylene oligomers on carbon nanotubes using physical vapor deposition
}

\author{
Lingyu $\mathrm{Li}^{\dagger}$, Yao Yang ${ }^{\ddagger}$, Guoliang Yang ${ }^{\ddagger}$, Xuming Chen $^{\S}$, Benjamin S. Hsiao ${ }^{\S}$, Benjamin $C h u^{\S}$, \\ Jonathan E. Spanier ${ }^{\dagger}$, Christopher Y. $\mathrm{Li}^{*}{ }^{\dagger}$
}

\footnotetext{
${ }^{\dagger}$ A. J. Drexel Nanotechnology Institute and Department of Materials Science and Engineering, Drexel University, Philadelphia, PA 19104

${ }^{\ddagger}$ Department of Physics, Drexel University, Philadelphia, PA 19104

${ }^{\S}$ Department of Chemistry, Stony Brook University, Stony Brook, NY 11794
}

To whom correspondence should be addressed: chrisli@drexel.edu

RECEIVED DATE (to be automatically inserted after your manuscript is accepted if required according to the journal that you are submitting your paper to)

CORRESPONDING AUTHOR FOOTNOTE: Christopher Li, 215-895-2083 (P); 215-895-6760

(F) chrisli@drexel.edu. 


\section{Experimental}

Purified HiPco SWNTs were purchased from Carbon Nanotechnologies Inc. Arch-discharged Multi-Walled Carbon Nanotubes (AD-MWNTs) were purchased from Aldrich and washed with 2.4 M nitric acid for 0.5 hrs. The resulting AD-MWNTs were then centrifuged, collected and dried in a vacuum oven. Linear polyethylene (PE, MFI = 12 g/10 min) and 1, 2 dichlorobenzene (DCB) were purchased from Aldrich and used as received.

CNTs were dissolved in DCB (0.01wt \%) and sonicated for $~ 2$ hrs before dispersing on transmission electron microscopy (TEM) grids using spin coating. A Bransonic 8510R-DTH ultrasonic cleaner with a nominal frequency of $44 \pm 6 \mathrm{kHz}$ at $250 \mathrm{~W}$ was used for ultrasonication. A small drop of CNT/DCB solution was first placed on the TEM grid. The spin speed and time were $3000 \mathrm{rpm}$ and 60s, respectively. These CNTs dispersed TEM girds or carbon coated cover glass were then coated by PE vapor using the physical vapor deposition method. The experimental setup is shown in Figure S1. $15 \mathrm{~cm}$ distance between the substrate and the basket in the vacuum evaporator was chosen. Vacuum was controlled to be $\sim 10^{-4}-10^{-5}$ torr. The TEM gird was shadowed with Pt/Pd before

TEM observation to enhance the contrast. TEM experiments were conducted using a JEOL-2000FX microscope with an accelerating voltage of $160 \mathrm{kV}$. High resolution TEM (HRTEM) experiments were carried out using a JEOL-2010F microscope with an accelerating voltage of $200 \mathrm{kV}$. AFM experiments was conducted using a Nanoscope IIIa atomic force microscope (AFM) (Digital Instruments/Veeco, Santa Barbara, CA), operated in tapping mode. Rectangular silicon nitride cantilevers (model TESPW, Digital Instruments/Veeco, Santa Barbara, 
CA) were used throughout the study.

\section{Surface functionalization of MWNT with octadecylamide}

MWNTs (synthesized using chemical vapor deposition method) were provided by Nanostructured and Amorphous Materials, Inc. The diameter of the MWNT is about 8-15 nm. Concentrated $\mathrm{H}_{2} \mathrm{SO}_{4} / \mathrm{HNO}_{3}$ Mixture (3:1) was used for oxidation. ${ }^{1,2}$ The oxidation generated numerous surface acidic groups such as carboxylic acid groups. The carboxylic acid groups reacted with amine at higher temperature in the next amidation reaction. ${ }^{2-4}$ For the amidation reaction, the oxidized MWNTs were dispersed in octadecylamine and maintained at $180-190{ }^{\circ} \mathrm{C}$ under nitrogen for 24 hours.

\section{References}

1. Esumi, K.; Ishigami, M.; Nakajima, A.; Swawda, K.; Honda, H. Carbon, 1996, 34, 279-281.

2. Sun, Y.; Fu, K.; Lin, Y.; Huang, W. Acc. Chem. Res. 2002, 35, 1096-1104.

3. Chen, X.; Yoon, K.; Burger, C.; Sics, I.; Fang, D.; Hsiao, B. S.; Chu B. Macromolecules 2005

38, 3883-3893.

4. Qin, Y.; Liu, L.; Shi, J.; Wu, W.; Zhang, Jun.; Guo, Z.-X.; Li, Y. Zhu, D. Chem. Mater. 2003, 15, 3256-3260. 


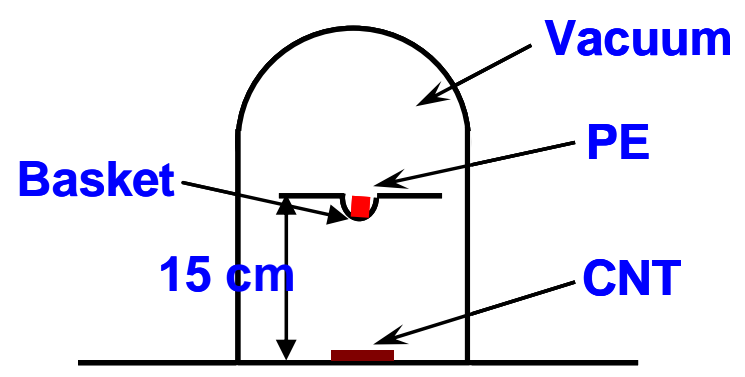

Figure S1. Schematic representation of the PVD experimental setup. CNTs were deposited on carbon coated glass slides before PVD.

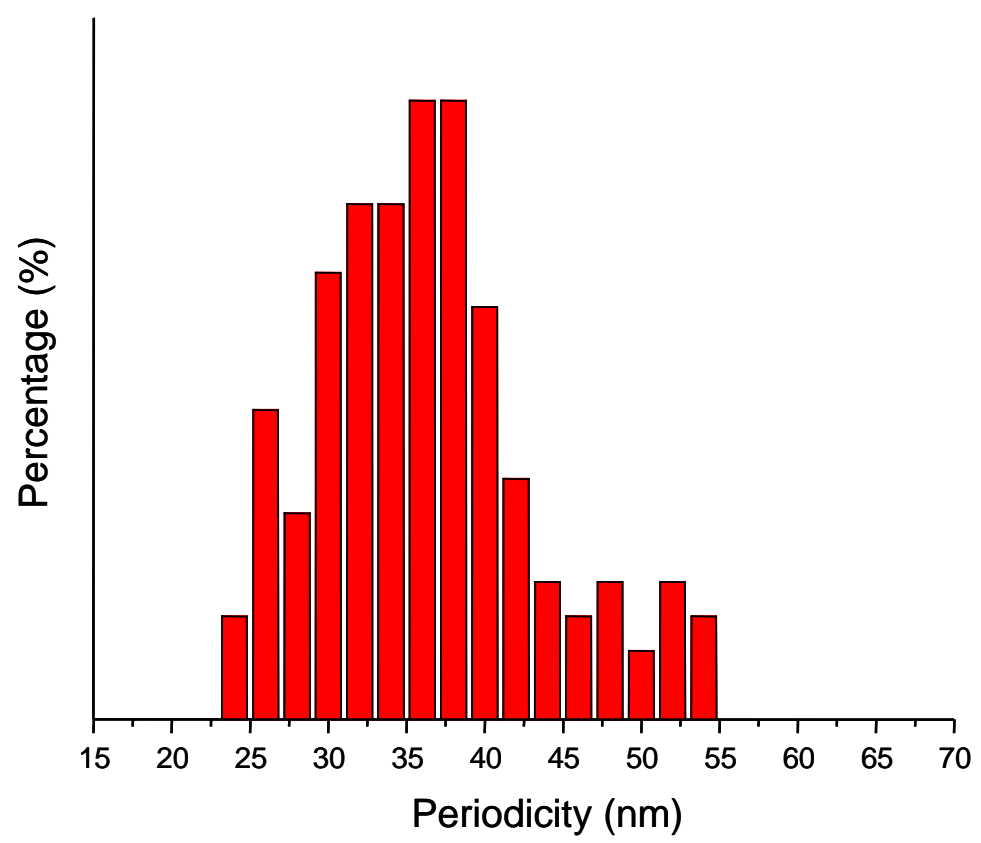

Figure S2. Histogram analysis of the periodicity of 2D NHSK. 136 data points were measured from TEM micrographs by using Scion Image. The average interval is $37.4 \mathrm{~nm}$ and standard deviation is $7.9 \mathrm{~nm}$. 


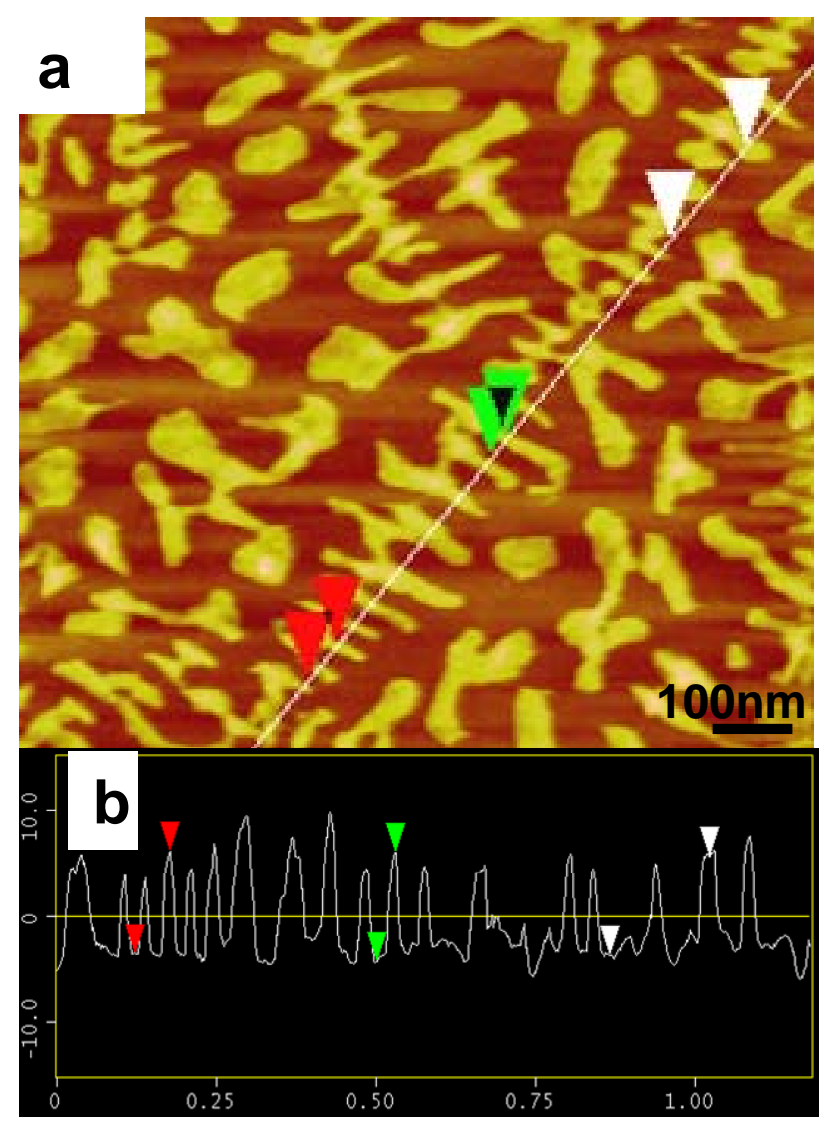

Figure S3. (a) AFM section analysis shows the vertical distances of the PE rods are 9.6, 10.2 and $9.2 \mathrm{~nm}$ respectively as indicated by red, green and white arrows. Note that the line is slightly off the 2D NHSK center. (b) shows the corresponding height profile. 


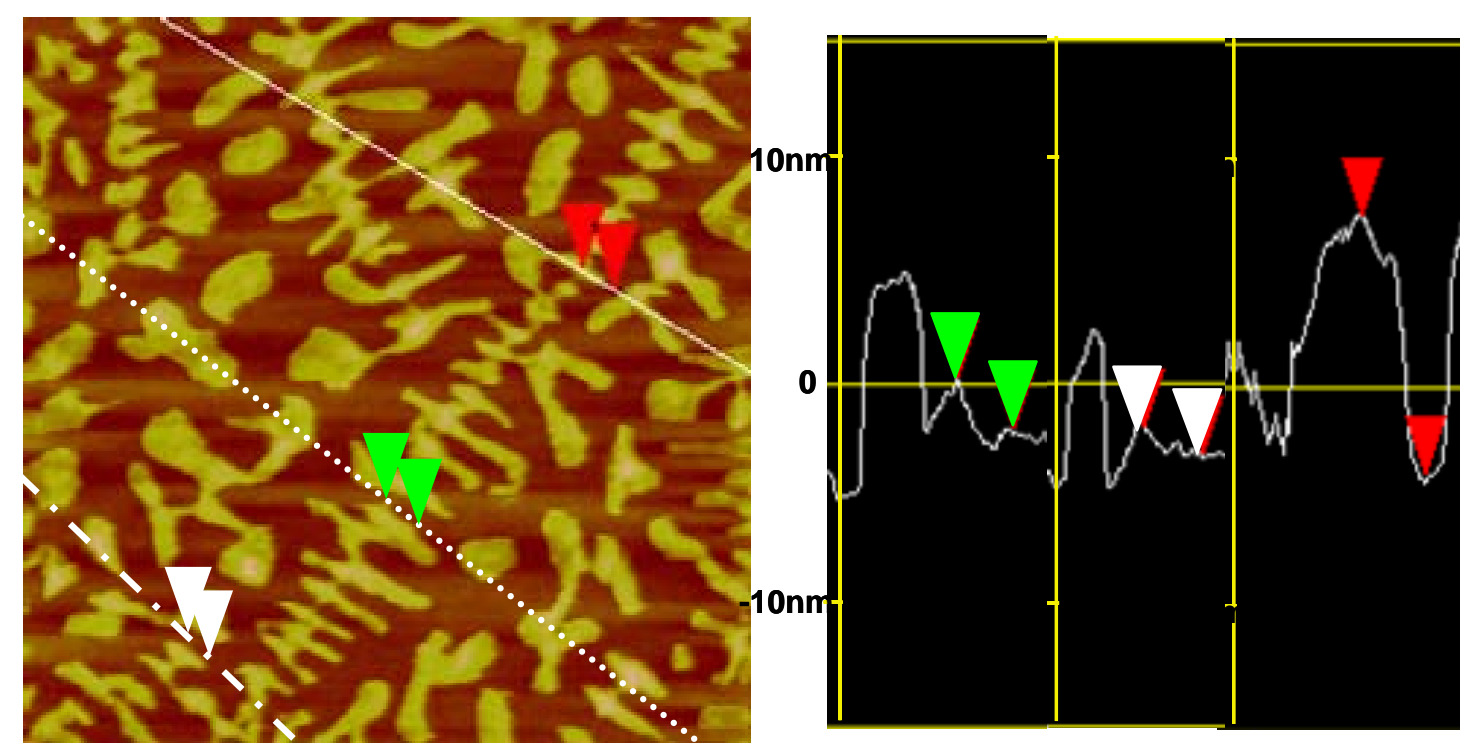

Figure S4. AFM section analysis shows vertical distances of $1.2 \mathrm{~nm}, 2.3 \mathrm{~nm}$ and $11.3 \mathrm{~nm}$ respectively indicated by white arrows, green arrows and red arrows. Note: $1.2 \mathrm{~nm}$ corresponds to the diameter of a single SWNT and $2.3 \mathrm{~nm}$ is probably due to formation of a small bundle of 2-4 SWNTs. Note that white arrows and green arrows indicate different 2D NHSKs. The height profile indicated by the red arrows shows that, at the center of the PE rod crystal, there is a small bump, which corresponds to the underneath SWNTs. 


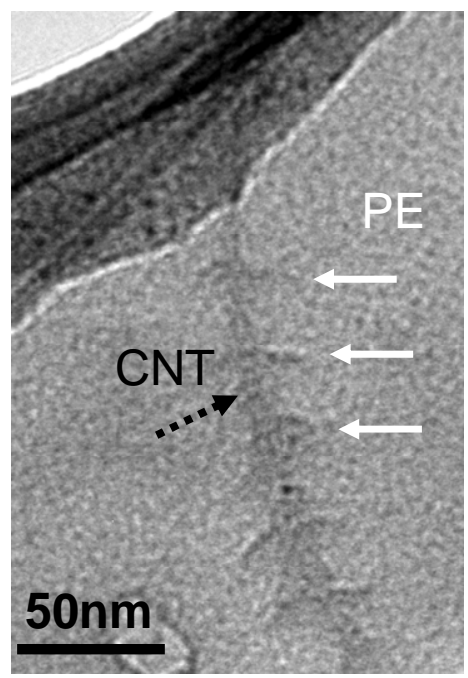

Figure S5. TEM image of a 2D NHSK on a lacy carbon grid. Note that the NHSK is on the carbon film area of the same grid of Figure $4 \mathrm{~b}$. The contrast is relatively low since the sample was not shadowed with $\mathrm{Pt} / \mathrm{Pd}$. 